\title{
Effect of Fed-batch vs. Continuous Mode of Operation on Microbial Fuel Cell Performance Treating Biorefinery Wastewater.
}

\author{
Tyler C. Pannell ${ }^{\mathrm{a}}$, R. Kannaiah Goud ${ }^{\mathrm{a}}$, Daniel J. Schell ${ }^{\mathrm{b}}$, Abhijeet P. Borole ${ }^{{ }^{\mathrm{a}, \mathrm{c}, \mathrm{d}}}$ \\ ${ }^{a}$ The University of Tennessee, Knoxville TN 37996. \\ ${ }^{\mathrm{b}}$ National Renewable Energy Laboratory \\ ${ }^{c}$ Biosciences Division, Oak Ridge National Laboratory, Oak Ridge, TN 37831-6226 \\ ${ }^{\mathrm{d} B r e d e s e n ~ C e n t e r ~ f o r ~ I n t e r d i s c i p l i n a r y ~ R e s e a r c h ~ a n d ~ E d u c a t i o n, ~ T h e ~ U n i v e r s i t y ~ o f ~}$ \\ Tennessee, Knoxville 37996. \\ *Corresponding author: borolea@ ornl.gov, Fax: (865) 5746442
}

\section{Notice of Copyright:}

This manuscript has been authored by UT-Battelle, LLC under Contract No. DE-AC0500OR22725 with the U.S. Department of Energy. The United States Government retains and the publisher, by accepting the article for publication, acknowledges that the United States Government retains a non-exclusive, paid-up, irrevocable, world-wide license to publish or reproduce the published form of this manuscript, or allow others to do so, for United States Government purposes. The Department of Energy will provide public access to these results of federally sponsored research in accordance with the DOE Public Access Plan (http://energy.gov/downloads/doe-public-access-plan).

C 2016. This manuscript version is made available under the Elsevier user license http://www.elsevier.com/open-access/userlicense/1.0/ 


\begin{abstract}
Bioelectrochemical systems have been shown to treat low-value biorefinery streams while recovering energy, however, low current densities and anode conversion efficiencies (ACE) limit their application. A bioanode was developed via enrichment of electroactive biofilm under fed-batch and continuous feeding conditions using corn stover-derived waste stream. The continuously-fed MFC exhibited a current density of $5.8 \pm 0.06 \mathrm{~A} / \mathrm{m}^{2}$ and an ACE of $39 \% \pm 4$. The fed-batch MFC achieved a similar current density and an ACE of 19.2\%, however, its performance dropped after 36 days of operation to $1.1 \mathrm{~A} / \mathrm{m}^{2}$ and $0.5 \%$, respectively. In comparison, the ACE of the continuously-fed MFC remained stable achieving an ACE of $30 \% \pm 3$ after 48 days of operation. An MFC treating a biorefinery stream post fuel separation achieved a current density of $10.7 \pm 0.1 \mathrm{~A} / \mathrm{m}^{2}$ and an ACE of $57 \%$ \pm 9 at an organic loading of $12.5 \mathrm{~g}$ COD/L-day. Characterization of the microbial communities indicate higher abundance of Firmicutes and Proteobacteria and lower abundance of Bacteriodetes and a higher level of Geobacter spp. (1.4\% vs. $0.2 \%$ ) in continuously-fed MFC vs. fed-batch MFC. The results demonstrate that limiting substrate to the equivalent maximum current that the anode can generate, maintains MFC performance over a long term for high strength wastewaters, such as those generated in the biorefinery.
\end{abstract}

Keywords: fermentation inhibitors, bioelectrochemical system, exoelectrogenic, biofilmforming, hydrolysate, corn stover. 


\subsection{Introduction}

The current density and coulombic efficiency $\left(\mathrm{CE}^{\#}\right)$ achieved during the conversion of the organic molecules in batch or fed-batch MFC systems is typically low, with a CE from very low to $50 \%$ [1-3]. Enrichment of bioanode under controlled substrate delivery (CSD) of continuous feeding conditions has been shown to improve the performance of MFCs [4]. This has been demonstrated with non-fermentable substrates such as acetate as well as fermentable substrates such as glucose, lactate, etc. [5-7]. The electrochemical conversion in bioanode depends on the anodophilic microorganisms, anode design and the operational parameters [4]. Conversion of complex wastewater streams typically requires use of microbial consortium containing numerous species of microorganisms. Eliminating growth of microbes such as methanogens, which lower Coulombic efficiency, requires understanding of the interactions between biofilm growth and the electrochemical processes. Recent work on the effect of shear [8], use of porous electrodes [7] and substrate delivery [5, 9] has provided important insights into improving the performance of bioelectrochemical systems (BESs). A combination of these factors has potential to improve performance of BESs treating real wastewaters. The ability to reach current densities up to $35 \mathrm{~A} / \mathrm{m}^{2}$ has been demonstrated using model substrates via formation of the electroactive biofilm under CSD conditions [9]. Similar methods need to be applied to real wastewaters to assess bioanode performance and determination of application potential.

The fermentation process for biofuel production from biomass has been undergoing rapid advances in the last decade resulting in commercial development of the biorefinery processes. An update of the process design analysis report released by National Renewable 
Energy Laboratory (NREL) in 2012 identified wastewater treatment costs on the order of $\$ 0.34$ per gallon of biofuel [10]. This was based on using anaerobic digestion technology for wastewater treatment which constituted $15.8 \%$ of the total operating cost of ethanol production. The wastewater contains energy in the form of organic matter which can potentially be recovered to improve overall process efficiency and economics of the process. This can add significant value to the biorefinery, if the energy products are directly usable in the biorefinery and have higher value than biogas. BESs can generate electricity, hydrogen or other value-added products from the wastewater [11, 12]. Conversion of the furan aldehydes including furfural and hydroxymethylfurfural as well as phenolic molecules to electricity using microbial fuel cells (MFCs) has been demonstrated [13]. The ability to remove the majority of the inhibitory molecules as well as fermentation byproducts resulting from conversion of corn stover hydrolyzate to ethanol in an MFC was also recently demonstrated [1].

In this study, we investigate enrichment of anode biofilm using a biorefinery waste stream under fed-batch and CSD or continuously-fed conditions. The feed stream was generated from an ethanol production process using corn stover as the feedstock. The organic content of the stream was high and required dilution to achieve desirable concentration in the feed to the anode. The effect of feeding the anode at high concentrations was studied in a fedbatch MFC. The feeding operation was controlled in a second MFC limiting it to the ability of the MFC to produce maximum current, determined via voltammetry. The hypothesis behind the investigation is that providing the substrate at a rate proportional to the bioanode's ability to produce current will favor enrichment of electroactive organisms, 
while minimizing growth of methanogens and other non-electrogens. The performance of the bioanode developed under CSD conditions was compared to the bioanode developed under fed-batch conditions. The microbial communities were characterized to understand the relationship between the composition of the community and MFC performance.

\section{Materials and Methods}

\subsection{Preparation of representative biorefinery wastewater stream}

A sample of a process stream representing biorefinery wastewater was generated at National Renewable energy Laboratory (NREL). Corn stover was used as the feedstock, which was pretreated, hydrolysed and fermented to produce ethanol. The details of the method are reported elsewhere [1]. Briefly, the corn stover was treated in a $5 \mathrm{~kg} / \mathrm{hr}$, pilotscale reactor using dilute sulfuric acid for $5 \mathrm{~min}$ at a solids loading of $30 \%$, followed by enzymatic hydrolysis and fermentation using a glucose-xylose fermenting bacterium, Zymomonas mobilis $8 \mathrm{~b}$ [14]. The batch was terminated after three days and the broth autoclaved to kill live bacteria. It was then filtered and vacuum distilled to remove ethanol and then shipped from NREL to Oak Ridge National Laboratory (ORNL). A sample of a fermentation broth which was not distilled was also used in a second set of MFC experiments. The samples are henceforth referred to as stover process water (SPW). The sample which was vacuum-distilled is referred to as SPW1, and the sample which was not distilled and contained the fermentation product, ethanol is referred to as SPW2. 


\subsection{Sample characterization}

The SPW samples were characterized via high performance liquid chromatography (HPLC) equipped with a refractive index and a UV-Vis detector (Hitachi LaChrome Elite System). Two different columns were used to separate and characterize the individual sugars, organic acids, furan aldehydes and phenolic compounds [1]. HPLC was also used to analyze samples after MFC treatment. A chemical oxygen demand (COD) analysis was conducted on the samples using a HACH kit (catalog \# 2125915). The lower limit of the COD analysis was $0.1 \mathrm{~g} / \mathrm{L}$, below which the analysis was not reproducible.

\subsection{MFC construction and inoculation}

The MFC used in this study consisted of a cylindrical carbon felt anode (projected surface area $=12.56 \mathrm{~cm}^{2}$, specific surface area $=454 \mathrm{~cm}^{2} \mathrm{~cm}^{-3}$ with anode volume $=15.96 \mathrm{~cm}^{3}$ ) separated from the cathode by a Nafion-115. An air cathode made of platinum-deposited carbon $\left(0.5 \mathrm{mg} / \mathrm{cm}^{2} ; 10 \% \mathrm{Pt}\right.$; E-TEC) was used. The experiments were carried out at room temperature $\left(22^{\circ} \mathrm{C}\right)$. The MFC was inoculated using a previously enriched exoelectrogenic consortium operating under fed-batch conditions [13]. The inoculum consisted of a $5 \mathrm{~mm}$ diameter $\mathrm{x} 12.5 \mathrm{~mm}$ length core cut out of the bioanode carbon felt. The inoculum was immediately transplanted into the similar-sized hole of new MFC. The organic matter in the SPW samples served as the carbon source for the MFC. The MFCs were investigated using two substrates: one with SPW1 as substrate (MFC1) and the other with SPW2 as the 
substrate (MFC2). The substrate solution was filtered through 0.45 micron filter prior to use in MFCs. An external resistance of $250 \Omega$ was used initially. The resistance was reduced in a step-wise manner as shown in Table 1 . The resistance was lowered until it reached a final value of $10.6 \Omega$ for MFC1 and $20 \Omega$ for MFC2. It was maintained at this final value for the rest of the experiments, except during linear sweep voltammetry measurements. A minimal nutrient medium consisting of mineral salts and vitamin solution was used as reported previously [15]. The medium was placed in an external reservoir and circulated through the anode chamber at a flow rate of $7 \mathrm{~mL} \mathrm{~min}^{-1}$. The growth of planktonic biomass was minimized after the first week by flushing it out of the anode with a syringe at high shear rate (flow rate of $>30 \mathrm{ml} \mathrm{min}^{-1}$ ). This procedure was performed every 2-3 days to promote biofilm formation and minimize accumulation of planktonic biomass, as reported previously $[6,15]$.

\subsection{Continuous vs fed-batch operation of MFCs.}

After establishment of the anode biofilm, the MFCs were evaluated under continuously-fed conditions. MFC1 and MFC2 were operated under the continuous substrate delivery (CSD) mode of operation to assess current density and conversion efficiency. The substrate loading was increased gradually from 0 to $12.5 \mathrm{~g} \mathrm{COD/L-day} \mathrm{for} \mathrm{MFC1} \mathrm{and} \mathrm{from} 0$ to 13.7 g COD/L-day for MFC2 as shown in Table 1. All concentrations and loading rates are reported in terms of $\mathrm{g} \mathrm{COD} / \mathrm{L}$ or $\mathrm{g} \mathrm{COD} / \mathrm{L}-\mathrm{day}$, henceforth. A concentrated stock solution of substrate was added continuously into the flow line entering the MFC via a syringe pump. The performance of the MFCs was monitored continuously by measuring voltage 
across a resistive load. The CSD mode of operation represented controlled substrate supply. In a second experiment, substrate supply was not limited. The MFC \#2 was operated in a fed-batch mode using SPW2 as the substrate to examine the effect of substrate concentration on MFC performance, when it is not limited. In this fed-batch experiment, the MFC was fed at a concentration ranging from $1.3 \mathrm{~g} / \mathrm{L}$ to $130.9 \mathrm{~g} / \mathrm{L}$, at the beginning of the run and operated in a recirculation mode until the current dropped below $0.1 \mathrm{~mA}$. This allowed a comparison of the unlimited substrate supply (fed-batch mode) to controlled substrate supply (CSD mode). It was envisioned that the CSD mode would control excessive growth of the biofilm, particularly the growth of methanogens and give exoelectrogens and supporting fermentative community an opportunity to populate the large surface area offered by carbon felt.

\subsection{Electrochemical analyses}

\subsection{Voltammetry}

The power density of the MFCs was analyzed via linear sweep voltammetry (LSV) using a two electrode system controlled by a potentiostat (Reference 3000, Gamry, U.S.A.). The working electrode was anode and the reference and counter electrodes were both connected to the cathode, in a two electrode configuration. The scan was conducted from $-0.5 \mathrm{~V}$ to $+0.1 \mathrm{~V}$ at a rate of $1.2 \mathrm{mV} \mathrm{sec}^{-1}$. The power vs. current curve was generated by calculating the power at each point that showed positive current from anode to cathode using the following formula:

Power $=\mathrm{I} * \mathrm{~V}$ 
The power density and current density were calculated by normalizing with electrode projected area, which was $12.56 \mathrm{~cm}^{2}$. Maximum current production was analyzed via voltammetry using a three-electrode system. The working electrode was anode, counter electrode was cathode and the reference electrode was $\mathrm{Ag} / \mathrm{AgCl}$ electrode $(\mathrm{BASi}$, West Lafayette, IN). The analysis was conducted after the current output was stable for a period of 24 hours for each condition. The sweep was conducted in anodic as well as cathodic direction and the cathodic current density is reported as a function of the applied potential. The scan was conducted from $-0.5 \mathrm{~V}$ to $+0.1 \mathrm{~V}$ at a rate of $1.2 \mathrm{mV} \mathrm{sec}^{-1}$.

\subsection{Anode conversion efficiency and Coulombic efficiency determination}

Coulombic efficiency (CE) was calculated using the following formula:

$$
C E_{\text {anode }}=\frac{I_{o b s} t}{n_{s} n_{e} F}
$$

Where $I_{o b s}$, is the produced current, $\mathrm{n}_{\mathrm{s}}$ is the moles of substrate used and $n_{e}$, is the number of electrons available per mol of substrate (based on complete conversion to $\mathrm{CO}_{2}$ ), $\mathrm{F}$ is Faraday constant and $t$ is the duration of the experiment. The calculation of CE for the fedbatch experiment was based on measured COD for the samples, however, COD analysis of the samples from continuously-fed MFCs did not show detectable organic content. The COD for most of these samples from the CSD runs was below the detection limit, which was $0.1 \mathrm{~g} / \mathrm{L}$. Therefore, overall anode conversion efficiency (ACE) was calculated for the CSD experiments. This is defined as follows:

$$
A C E=\frac{I_{o b s} t}{n_{S T} n_{e} F}
$$


Where $\mathrm{n}_{\mathrm{sT}}$ is the total moles of substrate supplied during the experiment. In order to compare the results with fed-batch runs, ACE was also calculated for the fed-batch runs in addition to $\mathrm{CE}$ determination for these runs based on COD analysis, to allow comparison of the two modes of operation.

\subsection{Microbial community analysis}

A biofilm sample was collected and characterized via 16S rRNA analysis to determine the composition of the microbial community. The samples were collected from the anode on day 83 by dislodging the cells from the electrode using a hypodermic needle, followed by withdrawal of the cells using a syringe from the exit of the MFC anode. Analysis of samples collected from the fed-batch experiment was also conducted to compare with the community developed under CSD conditions. A similar method was used to collect biofilm sample from the batch-operated MFC. Genomic DNA was isolated using the standard freeze-thaw procedure, followed by phenol-chloroform extraction [16]. The gDNA was submitted to GENEWIZ, Inc. for sequencing. The v4 region of $16 \mathrm{~S}$ rRNA gene was amplified from the total community genomic DNA and sequenced using the Illumina MiSeq platform yielding $115 \mathrm{MB}$ of raw data. The raw sequencing data were uploaded to MG-RAST and after quality control the overlapping paired-end reads were joined together. The MG-RAST ID is 4567311.3. The dataset contained 91,256 sequences totaling 578,455 base pairs with an average length of 289 bps. The summary for this upload set is as follows: 154 sequences $(0.8 \%)$ failed to pass the QC pipeline. Of the sequences that passed QC, 19,073 sequences (99.0\%) contain 16S rRNA genes. $29(0.2 \%)$ of the sequences that 
passed QC have no 16S rRNA genes. The $\alpha$-Diversity for the consortium from continuously-fed MFC was determined to be 55.1.

\section{Results and Discussion}

\subsection{Water sample analysis}

Fermentation of pretreated and enzymatically hydrolyzed corn stover generated at the pilotscale facility at NREL was used to prepare a representative biorefinery wastewater sample. The sample contained residual sugars, ethanol, acetate, sugar-degradation products as well as phenolic compounds originating from lignin-degradation. Most of the glucose was converted into ethanol via fermentation, but xylose and other sugars were not completely converted, resulting in significant level of these sugars remaining in the SPW samples (Table 2). Furfural and HMF concentrations were low since both compounds are metabolized by Z. mobilis which was the microbe used in the fermentation process [17]. The SPW1 sample had a total organic content of $91.3 \mathrm{~g} / \mathrm{L}$ and the SPW2 contained 70.3 g/L. Analysis of the SPW samples for total organic determination revealed a COD of 130.9 $\mathrm{g} / \mathrm{L}$ for SPW1 and $83.3 \mathrm{~g} / \mathrm{L}$ for SPW2. Comparison of the COD obtained for the SPW samples with the total COD for compounds identified by HPLC analysis was conducted. This is reported in Supplementary Information, Table S1. The compounds identified by HPLC were determined to contribute about $45 \%$ to the total COD in the samples. The unaccounted COD was due to contributions of oligomeric sugars not identified by HPLC 
and other phenolic and lignin-derived compounds. Total COD measured via HACH analysis was used in determination of CE and ACE for the MFCs.

\subsection{Effect of loading rate on current production}

A microbial culture was established in the anode of MFC1 using SPW1 as the carbon and energy source. The MFC was operated in CSD mode after 24 hours of initial biofilm growth under batch conditions. The current production reached $0.8 \mathrm{~mA}\left(0.7 \mathrm{~A} / \mathrm{m}^{2}\right)$ by day 3 . Growth of the anode microbial consortium was followed with increase in current production over time. Figure 1 shows the power density obtained in MFC1 as a function of time. Maximum power density was obtained within 13 days of initiation of biofilm growth. The maximum current produced by the biofilm was determined using LSV vs. $\mathrm{Ag} / \mathrm{AgCl}$ reference electrode. Current produced at a poised potential of $-0.2 \mathrm{~V}$ was monitored and used as a measure of electroactivity. The substrate delivery rate was increased to match the maximum current produced by the biofilm and the MFC was operated at that loading for a fixed number of days to assess stability. The voltage generated by the MFC at decreasing resistances is shown in Figure 2A for one experiment at each loading, each of which lasted from 48 to 120 hours. Figure $2 \mathrm{~B}$ shows the current density generated during the runs, averaged for the individual runs. The average current density is plotted for all the runs conducted during the 75 day period. The current increased to $4 \mathrm{~mA}\left(3.2 \mathrm{~A} / \mathrm{m}^{2}\right)$ by day 10 , and remained stable at $3.8 \pm 0.2 \mathrm{~mA}$ at a substrate delivery rate of $4.9 \mathrm{~g} / \mathrm{L}$-day for 9 days Further increase in substrate delivery rate from $1.6 \mathrm{~g} / \mathrm{L}$-day to $12.5 \mathrm{~g} / \mathrm{L}$-day revealed continued increase in current production. The current density increased proportionally to 
the substrate delivery rate, reaching a maximum of $10.9 \mathrm{~A} / \mathrm{m}^{2}$. A voltammetric analysis was conducted by scanning the anode from -0.5 to $+0.1 \mathrm{~V}$ vs. $\mathrm{Ag} / \mathrm{AgCl}$ reference electrode for each of the loading rates. The maximum current density achieved during these runs was higher than that under chronoamperometric runs. The current was stabile above $-0.25 \mathrm{~V}$ vs. $\mathrm{Ag} / \mathrm{AgCl}$ for OLRs up to $4.9 \mathrm{~g} / \mathrm{L}$-day, but above this OLR, the current increasing continuously as scan potential was increased up to $+0.1 \mathrm{~V}$ (Supplementary Information, Figure S1). The maximum current density obtained at a potential of $-0.2 \mathrm{~V}$ was $14 \mathrm{~A} / \mathrm{m}^{2}$.

\subsection{Fed-batch vs. Continuously-fed MFC performance.}

Two additional MFCs were operated under different modes of operation: fed-batch and continuous feeding using the substrate SPW2. The anode biofilm was established to obtain a baseline current of $1 \mathrm{~mA}$ for both conditions, after which the mode of operation was changed to study the effect of substrate delivery on MFC performance. The bioanode from both MFCs were acclimated to the substrate SPW2 at a concentration of $1.3 \mathrm{~g} / \mathrm{L}$, prior to studying the effect of mode of operation.

In the MFC operated under CSD conditions, the nutrient medium was changed to a fresh medium and the porous anode was flushed with fresh medium to remove planktonic cells from the anode. The removal of the unattached cells did not change the current density, even though the planktonic biomass was removed from the anode. The MFC was operated at a loading of $6.8 \mathrm{~g} / \mathrm{L}$-day for 28 days and then at $13.7 \mathrm{~g} / \mathrm{L}-$ day for the next 15 days. Production of current proportional to the organic loading was observed, similar to MFC1 
operated under CSD conditions, although the rate of change was different. This may be due to the differences in biodegradability of the two substrates, SPW1 and SPW2, which contain different concentrations of the sugar oligomers, phenolic compounds and ethanol (Table 2). A current density of $3.7 \pm 0.11 \mathrm{~A} / \mathrm{m}^{2}$ was obtained at the organic loading of 6.8 g/L-day, and $5.7 \pm 0.06 \mathrm{~A} / \mathrm{m}^{2}$ at $13.7 \mathrm{~g} / \mathrm{L}$-day (Figure $3 \mathrm{~A}$ ). The direct proportionality between current and organic loading demonstrated that current production was substratelimited, as was observed in MFC1 under CSD conditions. Previous studies with model substrates using the same MFC design and CSD conditions have revealed similar results [6].

In a second MFC, the substrate was provided in a fed-batch manner. The MFC was fed at an initial concentration ranging from $1.3 \mathrm{~g} / \mathrm{L}$ to $130.9 \mathrm{~g} / \mathrm{L}$, the latter representing the full strength biorefinery stream, SPW2. The current density increased initially up to a substrate concentration of $2.6 \mathrm{~g} / \mathrm{L}$, reaching $5.8 \mathrm{~A} / \mathrm{m}^{2}$ (Figure 3B). However, increasing the substrate concentration further did not increase the current density. On the contrary, the current density decreased as the substrate concentration was increased. It dropped to $1.1 \mathrm{~A} / \mathrm{m}^{2}$ as the substrate concentration was increased to $130.9 \mathrm{~g} / \mathrm{L}$. The decrease in current density with increasing substrate concentration was not due to inhibition as demonstrated previously [1]. Operation of the MFC at a substrate concentration of $2.6 \mathrm{~g} / \mathrm{L}$ after a batch run at a concentration of $20.9 \mathrm{~g} / \mathrm{L}$ resulted in a current density of $3.7 \mathrm{~A} / \mathrm{m}^{2}$, which represented a drop of $38 \%$ compared to the previous run at same concentration, following the same downward trend as shown in Figure 3B. This decrease in current density at a lower substrate concentration implied that the reduction in current was potentially due to reasons other than inhibition. 


\subsection{Effect of operating mode on anode conversion efficiency}

Coulombic efficiency (CE) was determined for the fed-batch experiment as outlined in the Materials and Methods section. The CE decreased with increasing substrate concentration during fed-batch operation from $27.5 \%$ at $2.6 \mathrm{~g} / \mathrm{L}$ to $0.6 \%$ at $130.9 \mathrm{~g} / \mathrm{L}$. The COD could not be determined accurately for the continuously-fed MFCs due to the low substrate concentration used in these experiments. These experiments were conducted at a constant flow rate, while feeding the substrate into the flow line entering the anode. The maximum substrate concentration in the CSD experiments was $0.016 \mathrm{~g} / \mathrm{L}$. Use of low substrate concentrations has been shown to yield reasonable current densities corresponding to the organic loading rate used in experiments reported previously $[6,9,18,19]$. This is typically done by maintaining a high flow rate through the anode to account for the low concentrations.

In order to compare the continuously-fed MFCs with MFCs operated in the fed-batch mode, conversion efficiencies were determined using the total substrate fed into the anode, instead of the substrate converted in the anode. This is termed as anode conversion efficiency (ACE). ACE was determined for the fed-batch mode of operation as well and compared to the CE (Supplementary Information, Figure S2). The comparison shows that both parameters had a similar trend with respect to the substrate concentration used in the fedbatch experiment as well as time. The ACE for the fed-batch MFCs was compared with that of continuously-fed MFCs over the whole length of the experiments (Figure 4). The 
purpose of this comparison was to understand the changes in ACE with time for the continuously-fed MFCs. In both experiments, continuously-fed as well as fed-batch MFCs, the concentration/loading was increased with time. The results show that the ACE for the fed-batch experiment decreased with time, while that for the continuously-fed MFCs remained relatively stable (Figure 4). The relationship of ACE was also examined with respect to substrate loading or concentration (Figure S2 and S3). The ACE for MFC2 under fed-batch mode of operation ranged from $19.2 \%$ to $0.5 \%$ over the range of COD concentrations studied. The drastic drop in ACE for the fed-batch operation vs. the continuously-fed MFCs indicates that the fed-batch operation results in degradation of the MFC performance. The two modes of operation differed in the amount of substrate available for the microorganisms in the anode. The ACE for the continuously-fed MFCs remained at higher values for both substrates, SPW1 and SPW2. For MFC fed with SPW1, an average ACE of $57 \% \pm 9$ was obtained at the highest organic loading, while it was $30 \%$ \pm 3 for MFC using SPW2 as the substrate. Using the substrate SPW2 in fed-batch MFC resulted in the ACE dropping to $0.6 \%$ at the highest loading, which represented a condition of excess loading in the MFC. The results indicate that uncontrolled loading in MFCs under fed-batch conditions can lead to precipitous drop in performance.

Previous work conducted using model substrates, glucose and lactate in a continuously-fed MFC [9] was reported to result in a current density as high as $35 \mathrm{~A} / \mathrm{m}^{2}$ at anode poised potential of -0.2 vs. $\mathrm{Ag} / \mathrm{AgCl}$, with $\mathrm{CEs}$ ranging in the $60-80 \%$. The results obtained in the current study using the SPW1/SPW2 substrates demonstrate that controlling the substrate concentration and the mode of delivery can result in high current density and ACE using 
complex substrate mixtures as well. The results demonstrate that operation of the MFCs under controlled substrate delivery conditions can lead to higher electroactivity.

\subsection{Electro-active biofilm growth and the effect of substrate delivery mode}

MFCs inoculated with a microbial consortium and operated under fed-batch conditions have potential to grow electrogenic, fermentative, methanogenic or any other type of microbes capable of growing in an anoxic environment. The rate of growth of individual members of the community depends on the specific growth rate of each organism. It has been reported that electrogenic bacteria may have higher specific growth rate compared to acetogenic methanogens [20], however, it has been observed that in the presence of fermentable substrates, methanogens and fermentative bacteria can grow to be the dominant members of the community [21, 22]. A recent study reported that methanogenesis is limited when the substrate concentration used in the anode is low [19]. The products of the reactions occurring in the anode resulting from the combined metabolism can further influence the subsequent enrichment and population distribution, especially when long term growth experiments are conducted such as those typical of MFCs. If the substrate addition rate is controlled to match the current production rate, it may be possible to control growth of microbes which have higher growth rate than electrogens by combining the substrate delivery with periodic removal of planktonic organisms during enrichment, thereby preventing take-over of the anode consortia by nonelectrogenic microbes. In the experiments reported here, the substrate loading in continuously-fed MFCs was limited to a level corresponding to the maximum current that 
could be generated by the MFCs. For example, the maximum current density obtained at OLR of $4.9 \mathrm{~g} / \mathrm{L}$-day and a scan potential of $-0.2 \mathrm{~V}$ was $7.2 \mathrm{~A} / \mathrm{m}^{2}$ for MFC1 (Supplementary Information, Figure S1). Under load conditions, the current density obtained was only 4.5 $\mathrm{A} / \mathrm{m}^{2}$. The voltammetric analysis resulted in higher current because the anode was being poised at higher potentials that the half-cell potential of the anode reached during MFC operation, which was about $-0.35 \mathrm{~V}$ vs. $\mathrm{Ag} / \mathrm{AgCl}$ reference. On day 17 , the organic loading was set to the next higher rate of $6.3 \mathrm{~g} / \mathrm{L}$-day, which is equivalent to $7.6 \mathrm{~A} / \mathrm{m}^{2}$ at $100 \% \mathrm{CE}$. This allowed only incremental increase in substrate availability that enabled the biofilm to reach a higher current which it was capable of approaching at that particular developmental stage. This mode of operation led to the ability of the continuously-fed MFCs to maintain the conversion efficiency in the anode to a high value in the range of $44-66 \%$, as observed experimentally, while in MFCs where substrate concentration/delivery was not controlled, the efficiency of the microbial consortia to produce electrons or current vs. other products dropped. This has direct implications for development of bioanodes for improvement of CE in MFCs fed with complex mixture of fermentable substrates such as that being produced from biorefinery processes as well as many other industrial wastewaters. Using the enrichment methodology suggested here, it may be possible to build the biofilm further to achieve removal of COD at rates comparable to that of high rate anaerobic digesters $(\sim 20$ g/L-day). In this work, we demonstrated COD removal rate $>5 \mathrm{~g} / \mathrm{L}$-day, obtained for the substrate loading rate of $9.08 \mathrm{~g} / \mathrm{L}$-day with an ACE of 50-60\%. This indicates that high rates of COD removal can be potentially accomplished with simultaneous power generation at high current densities $\left(>10 \mathrm{~A} / \mathrm{m}^{2}\right)$ for complex industrial wastewater streams. 


\subsection{Microbial characterization}

Analysis of the microbial consortia developed in the fed-batch and continuously-fed MFCs revealed establishment of different communities. Phylogenetic analysis revealed a diverse bacterial community in the anodic biofilm developed under CSD conditions comprising of Firmicutes (52.0\%), Proteobacteria (20.0\%), Bacteroidetes (11.0\%) and a number of other phyla resulting in a total of 24 genera (clusters). Figure 5 shows the distribution of the microbial population in the anode in the continuously-fed and fed-batch MFCs. The phyologenetic distribution was established with a boot-strap neighbor-joining method and is shown in Figure 6 for the continuously-fed MFC. Based on the phylogenetic analysis of partial 16S rRNA gene sequences, it is clear that specific microbial communities were established in the two anodes.

Clostridia was the largest class comprising $52 \%$ of the anodic biofilm in the continuouslyfed MFC. The predominant species in this phylum were Clostridium sphenoides (10\%), Clostridium indolis (9.2\%), Robinsoniella peoriensis (6.7\%), Clostridium bolteae (4.16\%) and two strains of Eubacterium. Several strains of Robinsoniella were also observed which showed 99\% similarity with Robinsoniella peoriensis strain YRC3.

The second most dominant phylum in the continuously-fed MFC was Proteobacteria. Betaproteobacteria was present in the anode at $10.7 \%$, which included Rhodocyclaceae (5.3\%), Azospira sp. (2.8\%) and Acidovorax sp. (1.5\%). The Rhodocyclaceae sp. showed 99.6\% similarity to members of an MFC community enriched previously (FJ393124.1) [15]. 
The Azospira sp. showed $99 \%$ sequence identity to uncultured Azospira sp. clone MFC63G02, and the Acidovorax sp. showed $100 \%$ similarity with Acidovoraxcaeni strain T-X2D. Members of Azospira have also been reported in an MFC fed with municipal primary clarifier effluent as the substrate [23].

Members of Gammaproteobacterium included Pseudomonas sp. (1.7\%) and Klebsiella oxytoca (1.6\%). The former showed $99 \%$ similarity to Pseudomonas nitroreducens strain vtcrmnrcc1 and the latter showed $99.6 \%$ similarity to Enterobacter sp. NFY132. Presence of Pseudomonas sp. indicates mediated electron transfer may be occurring in the anode since these microorganisms are primarily able to transfer electrons via mediators such as pyocyanin and other phenazine compounds [24-26]. A small portion of Deltaproteobacteria were also present (1.4\%). This comprised a strain similar to Geobacter sulfurreducens KN400 (99\% sequence similarity). Presence of a Geobacter sp. similar to the KN400 strain indicates that direct electron transfer may be occurring in these biofilms as well.

The third most dominant phylum in the continuously-fed MFC was Bacteroides (11.4\%). This included two species similar to uncultured Bacteroides Clone MFC-B162-G01 $(6.40 \%)$ and MFC-2-L2 (2.2\%), and third species similar to Dysgonomonasgadei MFCEB6 (2.4\%). Uncultured bacteria accounted for $9.8 \%$, with $100 \%$ sequence identity of the partial 16S rRNA gene Desulfovibrio spp. Phyla Firmicutes, Bacteroidetes, and Proteobacteria have commonly been detected in MFC anode [15, 27, 28], however, the relatively high abundance (16.5\%) of well-known exoelectrogenic bacteria, such as 
Clostridium spp., Geobacter spp., Pseudomonas spp. in the continuously-fed MFC suggests potential for high electroactivity.

The microbial community in the fed-batch experiment consisted of predominantly Bacteroidia (44.9\%), followed by Firmicutes (39.9\%), Proteobacteria (9.7\%), Actinobacteria $(0.31 \%)$, Euryarchaeota $(0.01 \%)$ and uncultured bacteria $(4.6 \%)$. The Bacteroidia class dominated in the batch study, but was present only at $11 \%$ in the continuously-fed MFC. Members of Bacteroidia were previously proven to be dominant in a biogas plant. Their abundance was reported to increase during the anaerobic fermentation process [29]. The predominant species in this phylum were Bacteroides graminisolvens (13.9\%), Bacteroides rodentium (13.8\%), Bacteroides uniformis (13.9\%). The CSD appears to have favored the growth of Firmicutes since this family increased from $39.9 \%$ in the fed-batch MFC to $52 \%$ in the continuously-fed MFC. The second most dominant phylum in the fed-batch MFC was Firmicutes, present at 26.8\%. The specific players included Clostridium indolis (3.9\%), Clostridium sphenoides (7\%) and Clostridium propionicum (3\%). Significant changes were observed in the Clostridia between the fedbatch and the continuously-fed system with reductions in most genera except the one similar to C. propionicum. A significant increase in a strain of Robinsoniella peoriensis was also observed going from fed-batch (0.2\%) to continuously-fed $\operatorname{MFC}(6.7 \%)$,

The third most dominant phylum in the fed-batch MFC was Proteobacteria (9.72\%). This family included the following genera: Betaproteobacteria (1.8\%), two uncultured Comamonadaceae species (0.88\%) similar to Acidovorax sp. KR1_RH06 and Azospira 
oryzae (0.89\%) showing $100 \%$ similarity to uncultured Azospira sp. MFC-B162-D12. Members of Deltaproteobacterium (0.4\%) included uncultured Desulfovibrio sp. (0.20\%) and uncultured Geobacter sp. (0.2\%). The former showed $100 \%$ similarity to uncultured Geobacter sp. clone MFCUaja3-89. The population of Proteobacteria also increased, rising from $9.7 \%$ in the fed-batch mode to $20 \%$ in the continuously-fed MFC. In particular, Geobacter spp. increased from $0.01 \%$ in the fed-batch system to $1.38 \%$ under the continuous delivery mode.

\subsection{Relating the changes in microbial community to the mode of operation}

This study demonstrated a microbial community capable of efficiently converting organic substrates in biorefinery wastewater containing furan aldehydes, phenolics, organic acids, alcohol and unconverted sugars to electricity. Use of MFCs to remove the inhibitory sugar and lignin degradation products from synthetic wastewater was reported previously revealing high power densities, reaching up to $3700 \mathrm{~mW} / \mathrm{m}^{2}$ with a coulombic efficiency of $69 \%$ [13]. The anodic microbial consortium from that MFC [1], formed inoculum for the fed-batch MFC community. The fed-batch MFC, in turn was used later to inoculate for the continuously-fed MFC. The analysis of the community developed in MFC fed with biorefinery wastewater under CSD mode showed that bacteria capable of utilizing the complex wastewater can be enriched and improved for better electrochemical performance. The consortium is expected to consist of at least two functionally different groups, including a fermentative group converting the larger molecular weight furan, phenolic and sugar molecules to intermediates which can be used by a second, electrogenic group to 
produce electrons. For example, presence of Azospira sp. in the anode suggested potential for degradation of the phenols. Phenol oxidase activity has been reported in a number of Azospirillum sp. [31].

The effect of substrate availability has not been specifically studied in the literature, however, Firmicutes have been reported to be important members of the anode community in MFCs [32, 33-35]. Firmicutes were also found to be dominant in the anode community, when fermentable substrates such as glucose, lactate or cellulose were fed into the MFC $[36,37]$. Results showed that the microorganisms in the fed-batch experiment had higher diversity ( $\alpha$-diversity $=24.3$ ), as compared to the community that developed in the continuously-fed system $(\alpha$-diversity $=55.0)$.

While the results obtained in this study show that many species observed in the MFCs were previously identified in MFCs, it does not provide specific evidence revealing how or why the mode of substrate addition might have changed the population distribution as it did. This requires further study with individual strains to determine their functional role and the effect of the process parameters. The fed-batch MFC showed very low abundance of methanogens at $0.006 \%$, which remained at about the same level $(0.005 \%)$ in the continuously-fed system. It should be noted that the inoculum for the batch study was derived from an MFC which was subjected to periodic removal of planktonic cells over several months which may have resulted in low level of methanogens in the anode community to start with [13]. Nevertheless, this study shows that the mode of the substrate addition has important effects on the microbial diversity as well as the abundance of 
different species in the biofilm community. Continuous delivery of the substrate proportional to the maximum-current producing capability of the biofilm shows that the increase in electroactivity is linked to favorable growth of electroactive organisms in continuously-fed MFCs.

\section{Conclusions}

The performance of MFCs enriched and operated under fed-batch and continuously-fed condition was measured via electrochemical and microbial analysis. The current density of a continuously-fed MFC increased to $10.7 \mathrm{~A} / \mathrm{m}^{2}$ with increasing substrate loading rate up to $12.5 \mathrm{~g} / \mathrm{L}-$ day, while it dropped from $5.8 \mathrm{~A} / \mathrm{m}^{2}$ to $1.1 \mathrm{~A} / \mathrm{m}^{2}$ in a fed-batch MFC. The anode conversion efficiency also dropped in fed-batch MFC from $19.2 \%$ to $0.5 \%$, while it remained relatively stable in the range $30 \% \pm 3$ to $39 \% \pm 4$ in a continuously-fed MFC using the same substrate. An MFC fed with a different substrate, also from a biorefinery process stream, yielded an ACE reaching 57\% \pm 9 under the continuous feeding conditions. Comparison of the results from the CSD experiments with fed-batch MFC suggests a link between the substrate delivery mode and MFC performance. Microbial characterization showed that the bioanode operated under the CSD conditions increased in population of known exoelectrogens such as Geobacter. Additionally, the microbial community from the continuously-fed MFC showed higher abundance of Clostridia, Azospira and Acidovorax and Rhodocyclaceae, and a reduction in Bacteroidia and methanogens compared to the anode community present in the fed-batch MFC. The results obtained provide evidence for the hypothesis that limiting the substrate concentration by controlling the rate of substrate 
supply can improve the performance of the MFC. The electroactivity of the biofilm was shown to be maintained by providing substrate at a rate comparable to the ability of the biofilm to produce current. The change in the abundance of organisms in the consortia, along with high current density and anode conversion efficiency proves that controlling substrate provided to the anode can influence electroactivity of the anode via a change in the microbial community.

\section{Acknowledgements}

Financial support for TCP was obtained from ASTRO Program, administered by the Oak Ridge Associated Universities. ORNL is managed by UT-Battelle, Inc. via a contract \# DEAC05-00OR22725 for the U.S. Department of Energy. The authors acknowledge support from BioEnergy Technologies Office, Energy Efficiency and Renewable Energy. Partial support from the Laboratory Directed Research and Development Program of Oak Ridge National Laboratory (ORNL) is also acknowledged.

\footnotetext{
Abbreviations

$\mathrm{CV}$ : coulombic efficiency

ACE: Anode conversion efficiency

CSD: Controlled-substrate delivery

SPW1: Stover process water (vacuum-distilled)

SPW2: Stover process water (not distilled)
} 
COD: Chemical oxygen demand

LSV: Linear Sweep Voltammetry

\section{References}

[1] A.P. Borole, C. Hamilton, D. Schell, Conversion of residual organics in corn stoverderived biorefinery stream to bioenergy via microbial fuel cells, Environ Sci Technol., 47 (2013) 642-648.

[2] T. Sleutels, L. Darus, H.V.M. Hamelers, C.J.N. Buisman, Effect of operational parameters on Coulombic efficiency in bioelectrochemical systems, Bioresource Technology, 102 (2011) 11172-11176.

[3] S.B. Velasquez-Orta, E. Yu, K.P. Katuri, I.M. Head, T.P. Curtis, K. Scott, Evaluation of hydrolysis and fermentation rates in microbial fuel cells, Applied Microbiology and Biotechnology, 90 (2011) 789-798.

[4] A.P. Borole, G. Reguera, B. Ringeisen, Z.-W. Wang, Y. Feng, B.H. Kim, Electroactive biofilms: Current status and future research needs., Energy Environ. Sci., 4 (2011) 48134834.

[5] A.P. Borole, C.Y. Hamilton, T.A. Vishnivetskaya, Enhancement in energy conversion efficiency and current density of 3-dimensional MFC anodes using pre-enriched consortium and continuous supply of electron donors. , Bioresour. Technol., 102 (2011) 5098-5104.

[6] A.P. Borole, C.Y. Hamilton, T.A. Vishnivetskaya, D. Leak, C. Andras, Improving power production from acetate-fed microbial fuel cells via enrichment of exoelectrogenic organisms in continuous flow systems., Biochem. Eng. J., 48 (2009) 71-80.

[7] T. Sleutels, R. Lodder, H.V.M. Hamelers, C.J.N. Buisman, Improved performance of porous bio-anodes in microbial electrolysis cells by enhancing mass and charge transport, International Journal of Hydrogen Energy, 34 (2009) 9655-9661.

[8] T.H. Pham, N. Boon, P. Aelterman, P. Clauwaert, L. De Schamphelaire, P. Oostveldt, K. Verbeken, W. Verstraete, K. Rabaey, High shear enrichment improves the performance of the anodophilic microbial consortium in a microbial fuel cell, Microbial Biotechnol., 1 (2008) 487-496.

[9] O. Ichihashi, T. Vishnivetskaya, A.P. Borole, High-Performance Bioanode Development for Fermentable Substrates via Controlled Electroactive Biofilm Growth, ChemElectroChem, 1 (2014) 1940-1947.

[10] D. Humbird, R. David, L. Tao, C. Kinchin, D. Hsu, A. Aden, P. Schoen, J. Lukas, M. Olthof, M. Worley, D. Sexton, D. Dudgeon, Process design and economics for biochemical conversion of lignocellulosic biomass to ethanol - dilute-acid pretreatment and enzymatic hydrolysis of corn stover, National Renewable Energy Laboratory (NREL), Golden, Colorado, 2011.

[11] A.P. Borole, Improving energy efficiency and enabling water recycle in biorefineries using bioelectrochemical cells., Biofuels, Bioproducts \& Biorefining, 5 (2011) 28-36. 
[12] A.P. Borole, J. Mielenz, Estimating hydrogen production potential in biorefineries using microbial electrolysis cell technology Intl J. Hydrogen Energy, 36 (2011) 1478714795.

[13] A.P. Borole, J. Mielenz, T.A. Vishnivetskaya, C.Y. Hamilton, Controlling accumulation of fermentation inhibitors in biorefinery water recycle using microbial fuel cells, Biotechnol. Biofuels, 2 (2009) 7.

[14] A. Mohagheghi, N. Dowe, D. Schell, C. TYat-Chen, C. Eddy, M. Zhang, Performance of a newly developed integrant of Zymomonas mobilis for ethanol production on corn stover hydrolysate, Biotechnol. Lett., 26 (2004) 321-325.

[15] A.P. Borole, C.Y. Hamilton, T.A. Vishnivetskaya, D. Leak, C. Andras, J. MorrellFalvey, B.H. Davison, M. Keller, Integrating engineering design improvements with exoelectrogen enrichment process to increase power output from microbial fuel cells $\mathrm{J}$. Power. Sources, 191 (2009) 520-527.

[16] P.L. Bond, S.P. Smriga, J.F. Banfield, Phylogeny of microorganisms populating a thick, subaerial, predominantly lithotrophic biofilm at an extreme acid mine drainage site, Applied and Environmental Microbiology, 66 (2000) 3842-3849.

[17] M. Franden, P. Pienkos, M. Zhang, Development of a high-throughput method to evaluate the impact of inhibitory compounds from lignocellulosic hydrolysates on the growth of Zymomonas mobilis, J. Biotechnol., 144 (2009) 259-267.

[18] A.J. Lewis, S. Ren, X. Ye, P. Kim, N. Labbe, A.P. Borole, Hydrogen production from switchgrass via a hybrid pyrolysis-microbial electrolysis process, Bioresource Technology, 195 (2015) 231-241.

[19] T. Sleutels, S. Molenaar, A. ter Heijne, C.J. Buisman, Low Substrate Loading Limits Methanogenesis and Leads to High Coulombic Efficiency in Bioelectrochemical Systems, Microorganisms, 4 (2016) 1-11.

[20] H.S. Lee, C.I. Torres, B.E. Rittmann, Effects of Substrate Diffusion and Anode Potential on Kinetic Parameters for Anode-Respiring Bacteria, Environ. Sci. Technol., 43 (2009) 7571-7577.

[21] H.S. Lee, P. Parameswaran, A. Kato-Marcus, C.I. Torres, B.E. Rittmann, Evaluation of energy-conversion efficiencies in microbial fuel cells (MFCs) utilizing fermentable and non-fermentable substrates, Water Res., 42 (2008) 1501-1510.

[22] P. Parameswaran, H.S. Zhang, C.I. Torres, B.E. Rittmann, R. Krajmalnik-Brown, Microbial Community Structure in a Biofilm Anode Fed With a Fermentable Substrate: The Significance of Hydrogen Scavengers, Biotechnology and Bioengineering, 105 (2010) 69-78.

[23] S.i. Ishii, S. Suzuki, T.M. Norden-Krichmar, K.H. Nealson, Y. Sekiguchi, Y.A. Gorby, O. Bretschger, Functionally stable and phylogenetically diverse microbial enrichments from microbial fuel cells during wastewater treatment, PloS one, 7 (2012) e30495.

[24] K. Rabaey, W. Verstraete, Microbial fuel cells: novel biotechnology for energy generation, Trends Biotechnol., 23 (2005) 291.

[25] H. Rismani-Yazdi, A.D. Christy, S.M. Carver, Z. Yu, B.A. Dehority, O.H. Tuovinen, Effect of external resistance on bacterial diversity and metabolism in cellulose-fed microbial fuel cells, Bioresource Technology, 102 (2011) 278-283.

[26] V. Sharma, P. Kundu, Biocatalysts in microbial fuel cells, Enzyme and Microbial Technology, 47 (2010) 179-188. 
[27] K.J. Chae, M.J. Choi, J.W. Lee, K.Y. Kim, I.S. Kim, Effect of different substrates on the performance, bacterial diversity, and bacterial viability in microbial fuel cells, Bioresource Technology, 100 (2009) 3518-3525.

[28] T. Shimoyama, S. Komukai, A. Yamazawa, Y. Ueno, B.E. Logan, K. Watanabe, Electricity generation from model organic wastewater in a cassette-electrode microbial fuel cell, Applied Microbiology and Biotechnology, 80 (2008) 325-330.

[29] A. Hanreich, U. Schimpf, M. Zakrzewski, A. Schlüter, D. Benndorf, R. Heyer, E. Rapp, A. Pühler, U. Reichl, M. Klocke, Metagenome and metaproteome analyses of microbial communities in mesophilic biogas-producing anaerobic batch fermentations indicate concerted plant carbohydrate degradation, Systematic and applied microbiology, 36 (2013) 330-338.

[30] R.L. Smith, S.P. Buckwalter, D.A. Repert, D.N. Miller, Small-scale, hydrogenoxidizing-denitrifying bioreactor for treatment of nitrate-contaminated drinking water, Water research, 39 (2005) 2014-2023.

[31] V. Nikitina, E. Vetchinkina, E. Ponomareva, Y.V. Gogoleva, Phenol oxidase activity in bacteria of the genus Azospirillum, Microbiology, 79 (2010) 327-333.

[32] Y.F. Choo, J. Lee, I.S. Chang, B.H. Kim, Bacterial communities in microbial fuel cells enriched with high concentrations of glucose and glutamate, Journal of microbiology and biotechnology, 16 (2006) 1481.

[33] K. Chung, S. Okabe, Continuous power generation and microbial community structure of the anode biofilms in a three-stage microbial fuel cell system, Applied microbiology and biotechnology, 83 (2009) 965-977.

[34] S. Jung, J.M. Regan, Comparison of anode bacterial communities and performance in microbial fuel cells with different electron donors, Applied microbiology and biotechnology, 77 (2007) 393-402.

[35] D. Xing, S. Cheng, J.M. Regan, B.E. Logan, Change in microbial communities in acetate-and glucose-fed microbial fuel cells in the presence of light, Biosensors and Bioelectronics, 25 (2009) 105-111.

[36] A.P. Borole, C.Y. Hamilton, T.A. Vishnivetskaya, D. Leak, C. Andras, J. MorrellFalvey, M. Keller, B. Davison, Integrating engineering design improvements with exoelectrogen enrichment process to increase power output from microbial fuel cells, Journal of Power Sources, 191 (2009) 520-527.

[37] H. Rismani - Yazdi, A.D. Christy, B.A. Dehority, M. Morrison, Z. Yu, O.H. Tuovinen, Electricity generation from cellulose by rumen microorganisms in microbial fuel cells, Biotechnology and bioengineering, 97 (2007) 1398-1407. 
Tables

Table 1: Operating conditions for the MFC1 and 2 under CSD conditions. The MFCs were operated under batch conditions for the first few days to enable biofilm establishment and operated under CSD conditions beginning day 12.

\begin{tabular}{|c|c|c|c|c|}
\hline & \multicolumn{2}{|r|}{ MFC1 } & \multicolumn{2}{|r|}{ MFC2 } \\
\hline Day & $\begin{array}{c}\text { Resistance, } \\
\mathbf{\Omega}\end{array}$ & $\begin{array}{c}\text { Organic loading rate, } \\
\text { g/L-day }\end{array}$ & $\begin{array}{c}\text { Resistance, } \\
\mathbf{\Omega}\end{array}$ & $\begin{array}{c}\text { Organic loading rate, } \\
\text { g/L-day }\end{array}$ \\
\hline 0 & & Start-up period & & \\
\hline 1 & 250 & $\begin{array}{c}\text { Start continuous } \\
\text { feeding of SPW1, } 1.18\end{array}$ & 250 & \\
\hline 2 & 100 & & 100 & \\
\hline 5 & & & 50 & a. \\
\hline 6 & & 2.35 & & \\
\hline 7 & 53 & & & \\
\hline 10 & & 2.34 & & \\
\hline 12 & & & & $\begin{array}{c}\text { Continuous feeding of } \\
\text { SPW2, } 3.0\end{array}$ \\
\hline 13 & 21 & & & \\
\hline 14 & & & 20 & \\
\hline 20 & & 3.53 & & \\
\hline 21 & 10.6 & & & \\
\hline 40 & & & & 6.0 \\
\hline 59 & & 6.81 & & \\
\hline 63 & & 9.08 & & \\
\hline
\end{tabular}




\begin{tabular}{|c|c|c|c|c|c|c|c|c|c|c|c|c|}
\hline $\begin{array}{l}\text { Concentrat } \\
\text { ion, } \mathrm{g} / \mathrm{L}\end{array}$ & Cellobiose & Glucose & Xylose & Arabinose & Xylitol & $\begin{array}{l}\text { Lactic } \\
\text { acid }\end{array}$ & Glycerol & $\mathrm{EtOH}$ & $\begin{array}{l}2- \\
\text { Furfural }\end{array}$ & HMF & $\begin{array}{l}\text { Acetic } \\
\text { acid }\end{array}$ & Other* \\
\hline SPW1 & 0.8 & 0.2 & 15.5 & 6.6 & 0.4 & 0.6 & 0.8 & 0.4 & 0.0042 & 0.004 & 9.0 & 36 \\
\hline SPW2 & 0.94 & 0.36 & 20.28 & 6.45 & NA & NA & NA & 25.98 & 0.04 & 0.009 & 8.7 & 25.1 \\
\hline \multicolumn{13}{|c|}{$\begin{array}{l}\text { * The concentration of 'other' components in the } \\
\text { carbohydrates and other components of biomass. } \\
\text { sample contained } 66.4 \mathrm{~g} / \mathrm{L} \text { total organic content. }\end{array}$} \\
\hline
\end{tabular}




\section{Figures}

Figure 1: Power density of MFC fed with SPW1 under continuous substrate delivery conditions.

Figure 2: Effect of substrate loading rate on MFC performance. The top section shows voltage raw data as a function of time for discrete time spans corresponding to individual runs at various OLRs. The lower section shows current density using average voltage for the individual runs, but for all tests conducted during the 75 day period. The spikes were not included in calculating the average. The MFC was fed with SPW1 continuously after an initial batch operation of 1 day.

Figure 3. Current density as a function of substrate loading rate for MFCs operated under CSD conditions (A) and fed-batch conditions (B). The blue diamonds represent current density obtained for MFC 1, while the red squares represent the current density obtained for MEC2, using SPW2 as the substrate.

Figure 4: Changes in coulombic efficiency under CSD operation conditions over a 73 day period. The results from the CSD experiment are compared with CE reported under batch conditions previously. The precipitous drop in CE in batch experiment conditions shows the inefficient conversion of substrates to electricity, potentially due to growth of non- 
exoelectrogenic bacteria in the anode under initial high substrate concentrations representative of batch operation.

Figure 5: Microbial characterization of anode consortia resulting from MFCs fed under (A) continuous substrate delivery conditions (B) and fed-batch mode.

Figure 6: Phylogenetic tree of the anode microbial consortia enriched under continuous substrate delivery conditions. Underlined names indicate an exoelectrogenic origin.

\section{Figures:}

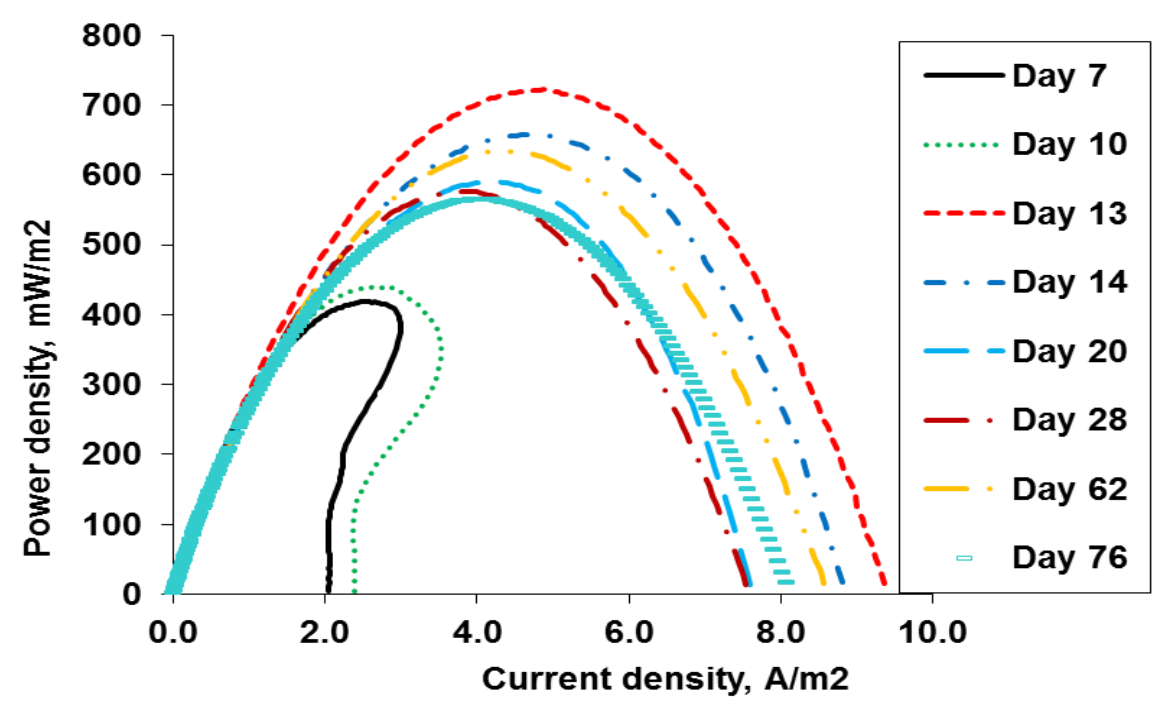

Figure 1: Power density of MFC fed with SPW1 under continuous substrate delivery conditions. 
A.

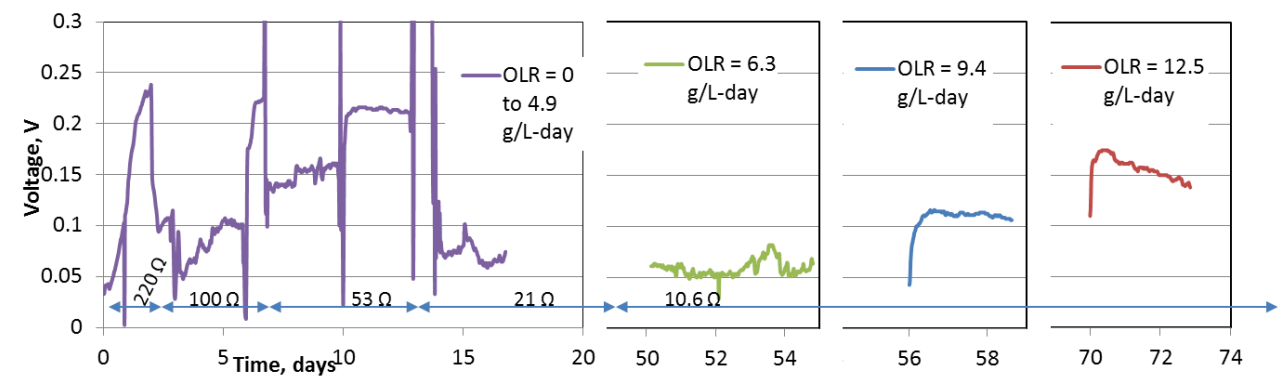

B.

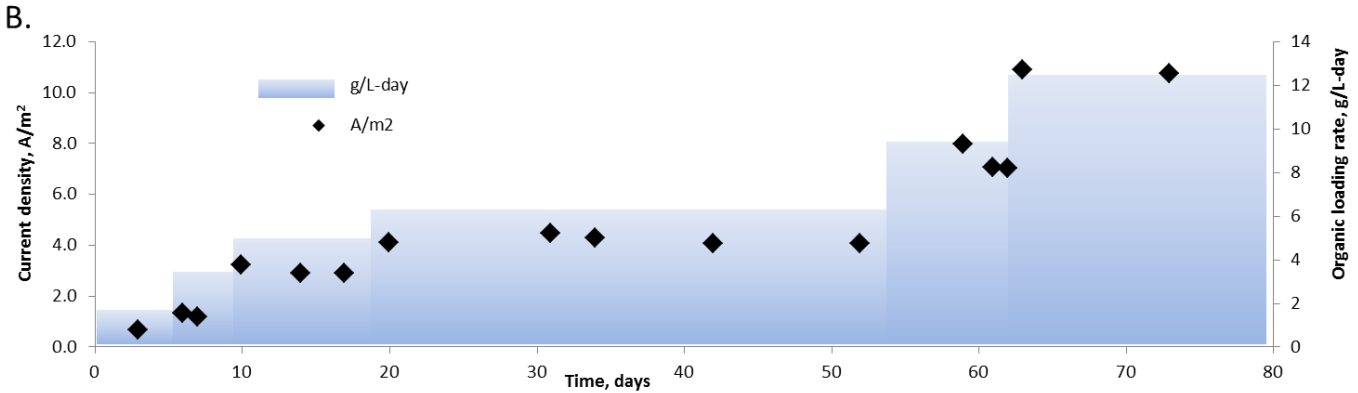

Figure 2: Effect of substrate loading rate on MFC performance. The top section shows voltage raw data as a function of time for discrete time spans corresponding to individual runs at various OLRs. The lower section shows current density using average voltage for the individual runs, but for all tests conducted during the 75 day period. The spikes were not included in calculating the average. The MFC was fed with SPW1 continuously after an initial batch operation of 1 day. 
A.

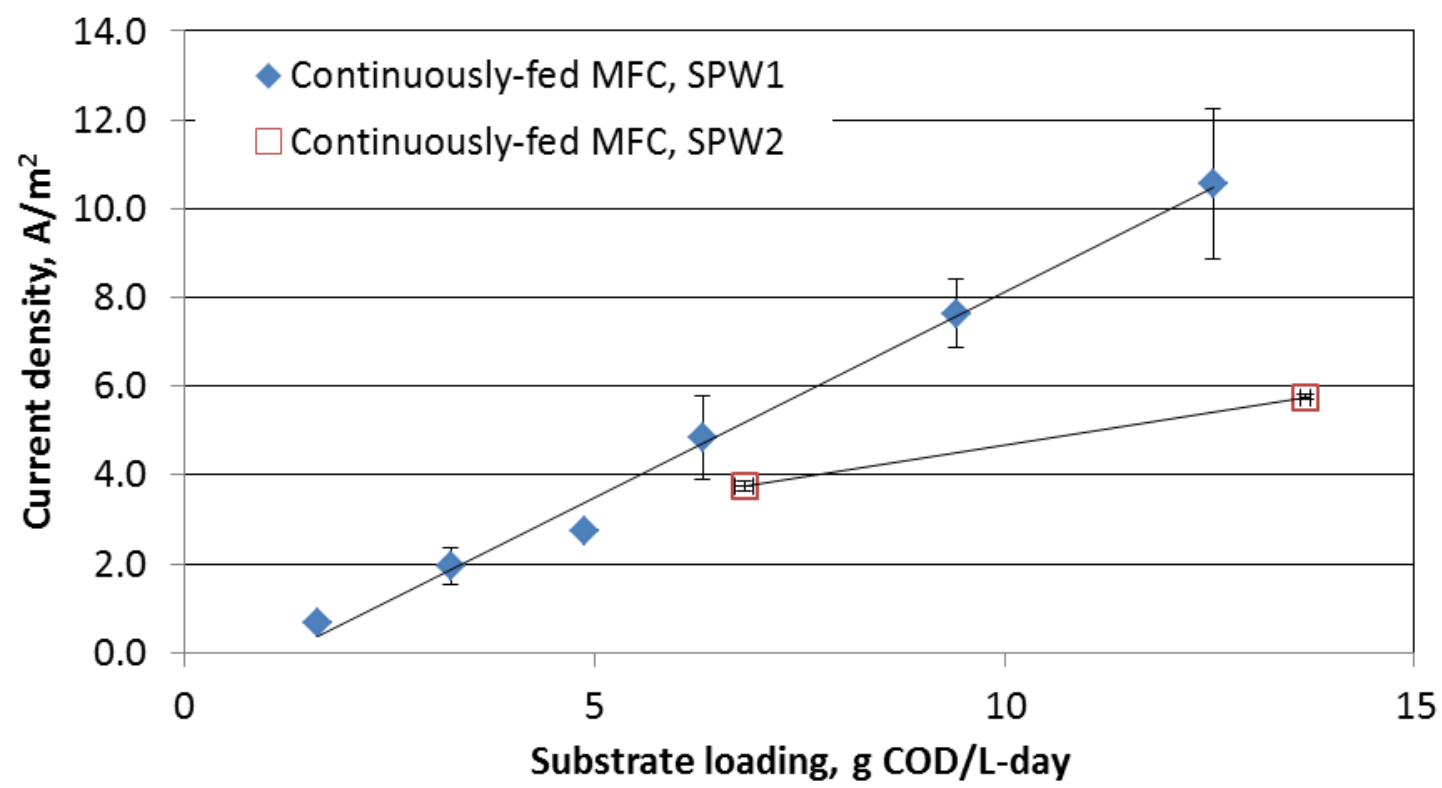

B.

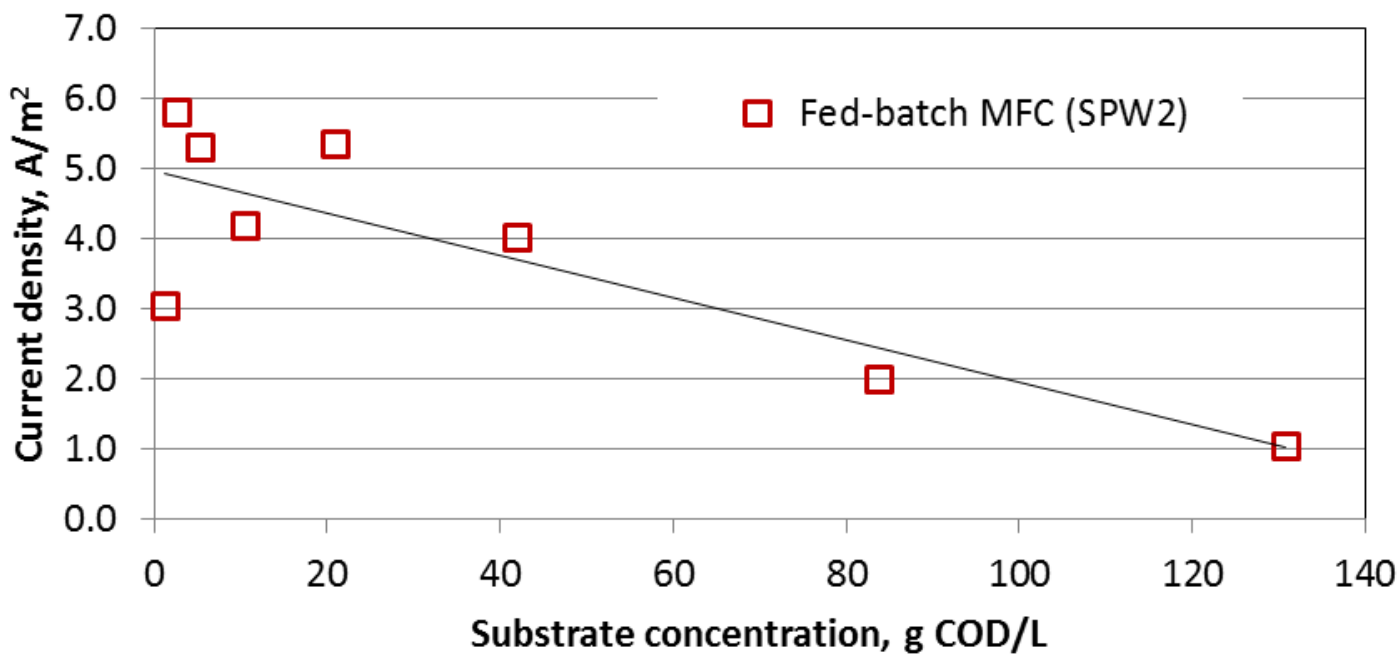

Figure 3. Current density as a function of substrate loading rate for MFCs operated under CSD conditions (A) and fed-batch conditions (B). The blue diamonds represent current density obtained for MFC 1, while the red squares represent the current density obtained for MEC2, using SPW2 as the substrate. 


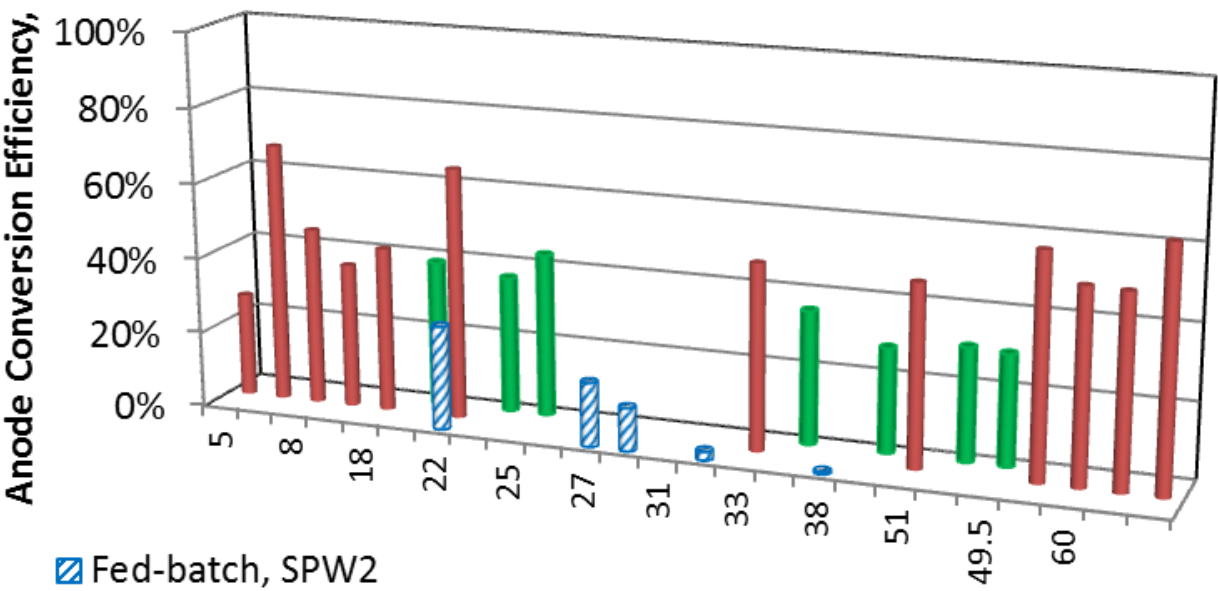

$\square$ Fed-batch, SPW2

Continuously-fed, SPW1

Time, days

Continuously-fed, SPW2

Figure 4: Changes in coulombic efficiency under CSD operation conditions over a 73 day period. The results from the CSD experiment are compared with CE reported under batch conditions previously. The precipitous drop in CE in batch experiment conditions shows the inefficient conversion of substrates to electricity, potentially due to growth of nonexoelectrogenic bacteria in the anode under initial high substrate concentrations representative of batch operation. 


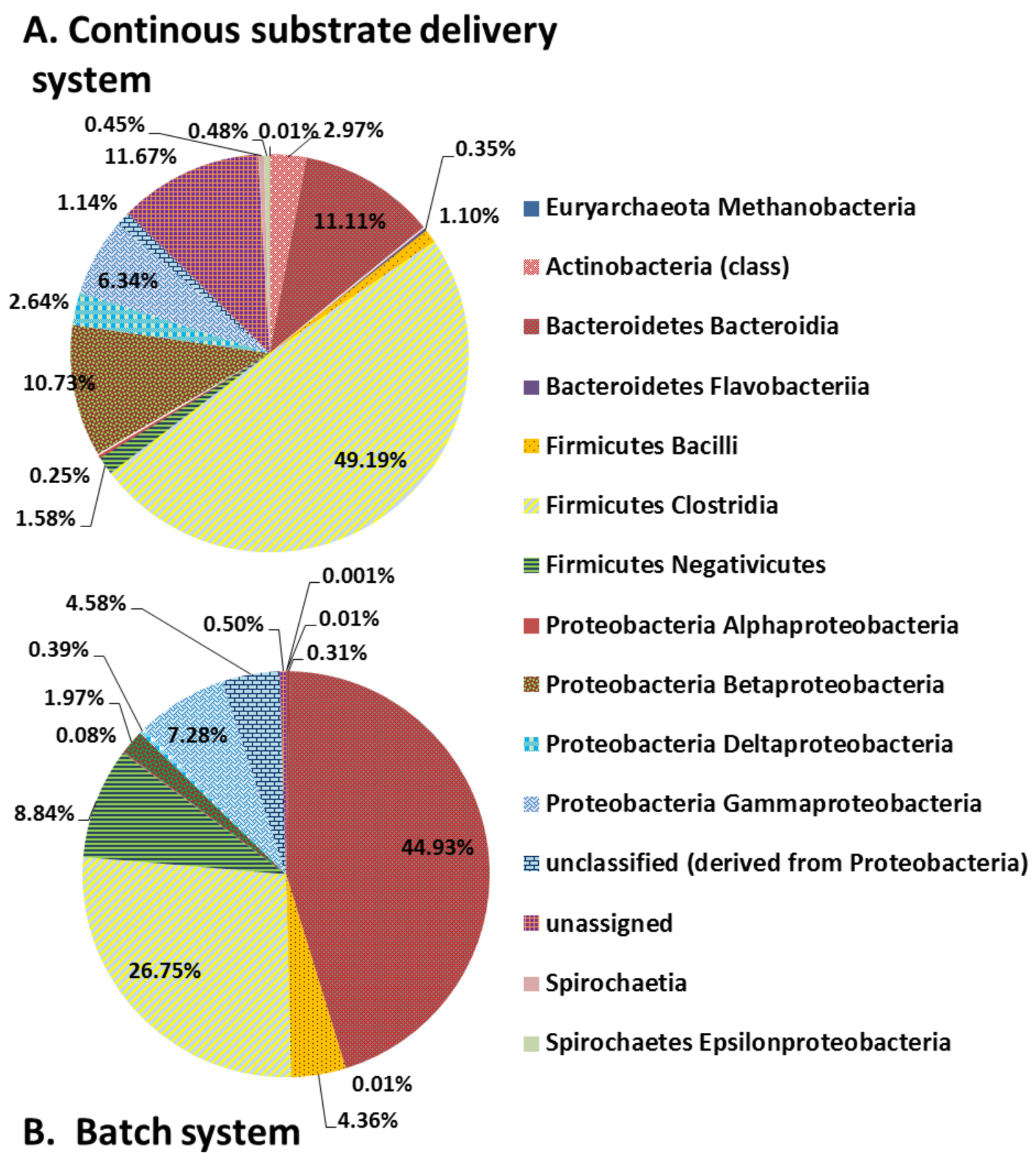

Figure 5: Microbial characterization of anode consortia resulting from MFCs fed under (A) continuous substrate delivery conditions (B) and fed-batch mode. 


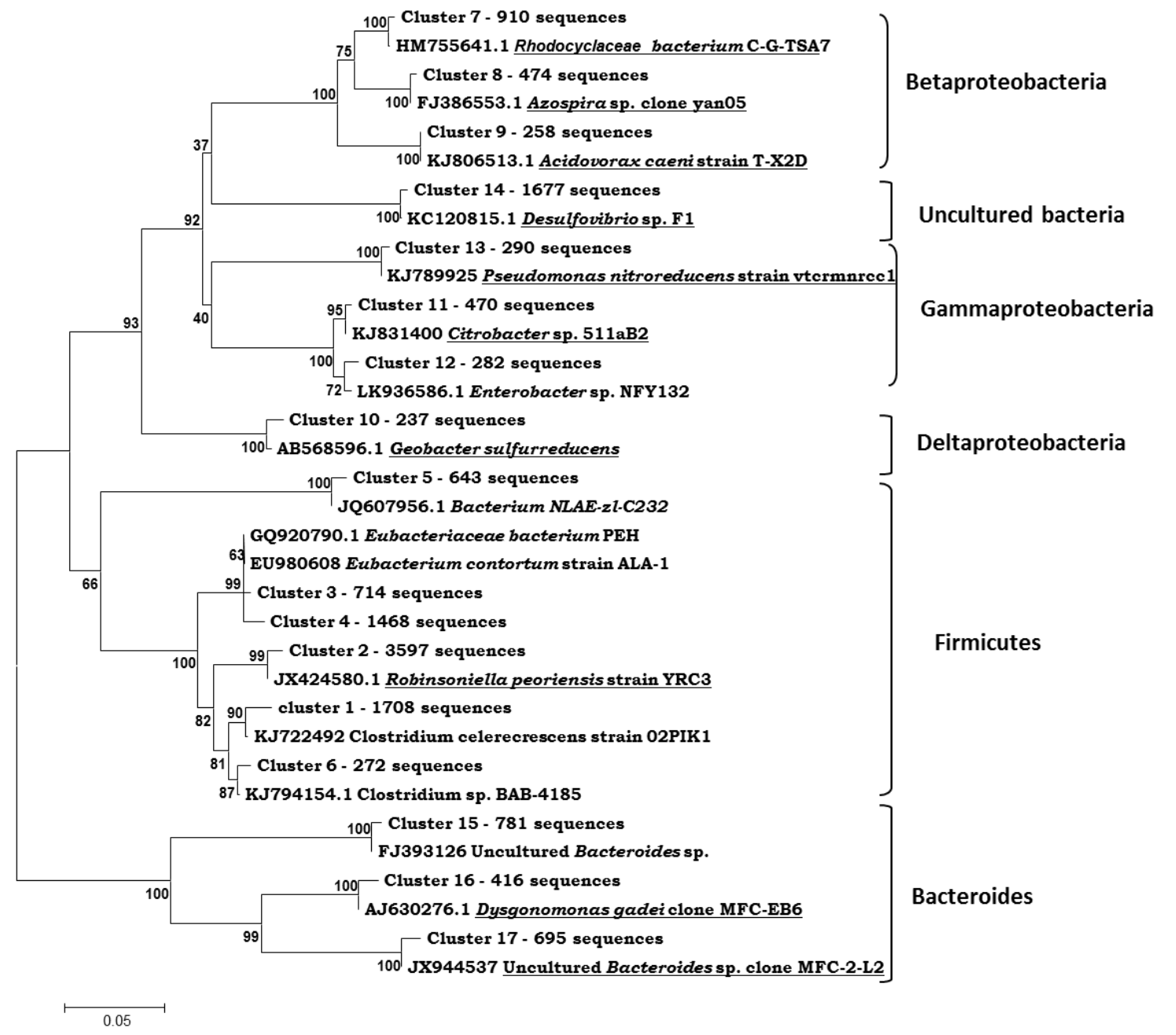

Figure 6: Phylogenetic tree of the anode microbial consortia enriched under

continuous substrate delivery conditions. Underlined names indicate an exoelectrogenic origin. 